\title{
FRIEDMAN'S MONEY SUPPLY RULE VS. OPTIMAL INTEREST RATE POLICY
}

\author{
George W. Evans* and Seppo Honkapohja**
}

\begin{abstract}
Using New Keynesian models, we compare Friedman's k-percent money supply rule to optimal interest rate setting, with respect to determinacy, stability under learning and optimality. First we review the recent literature: open-loop interest rate rules are subject to indeterminacy and instability problems, but a properly chosen expectations-based rule yields determinacy and stability under learning, and implements optimal policy. We show that Friedman's rule also can generate equilibria that are determinate and stable under learning. However, computing the mean quadratic welfare loss, we find for calibrated models that Friedman's rule performs poorly when compared to the optimal interest rate rule.
\end{abstract}

\section{INTRODUCTION}

The recent literature on monetary policy has focused on policy rules in which the interest rate is the chosen policy instrument, and a major finding is that the form of the interest-rate rule is crucial for inducing key desirable properties of the economy. For example, setting the interest rate based only on exogenous fundamental variables leads to instability problems if in fact private agents do not a priori have rational expectations (RE) but instead form expectations using standard adaptive learning rules. This was recently demonstrated by (Evans and Honkapohja, 2003b) in the context of the New Keynesian model that has become a standard framework in recent research on monetary policy. ${ }^{1}$ Another difficulty with such interest-rate rules is that they imply indeterminacy of rational expectations equilibria (REE). In other words, there exist other REE near the 'fundamental' REE, which can depend on extraneous factors solely through private expectations, see e.g. (Bernanke and Woodford, 1997) and (Woodford, 1999b). (Evans and Honkapohja, 2003a) provide a survey of the recent literature on learning, determinacy and monetary policy.

Interest-rate rules that react only to observable exogenous variables can be viewed as 'open-loop' policies, since they do not respond to variables that are

*University of Oregon

**University of Helsinki and the Bank of Finland

${ }^{1}$ (Howitt, 1992) raised earlier the same concern, but did not employ the New Keynesian model. 
endogenous to the economy. Making the interest rate depend on lagged endogenous variables, including possibly the lagged interest rate itself, may or may not provide a remedy to these problems. On this point see Evans and Honkapohja (2002a) for the case of optimal monetary policy under commitment and Bullard and Mitra $(2001,2002)$ for the case of instrument (or Taylor) rules. Evans and Honkapohja (2002a, 2003b) have argued that interest-rate setting should react to private forecasts of the endogenous variables, i.e. to inflation and output gap forecasts. Evans and Honkapohja (2002a) show that a reaction function of this type, with appropriately chosen parameters, can implement the optimal policy under commitment in a way that ensures both stability under learning and determinacy of the desired solution. In this paper we first review the results for this 'expectations-based' policy rule.

Our recommended implementation of optimal policy is, by its nature, a 'closed-loop' policy that requires considerable information. In particular, our policy rule depends on obtaining accurate measurements of both private expectations and exogenous shocks, and is based on a correct specification of the structural model and known values of key structural parameters. ${ }^{2}$ These demanding requirements suggest that it may be worth considering alternative open-loop policies. Are all open-loop policies subject to indeterminacy and learning instability? If these problems can be avoided, how satisfactory are these alternative policies in terms of achieving the policy objectives? To investigate this issue we here focus on a venerable, simple open-loop policy, namely Friedman's $k$-percent money supply rule. This rule was initially suggested by Friedman $(1948,1959)$ in part as a way to cope with parameter and model uncertainty.

Our results are easily summarized. Based on numerical calculations for calibrated New Keynesian models, we find that the Friedman $k$-percent rule appears to induce both determinacy and stability under learning. Thus this openloop money supply rule does meet some key requirements for a desirable monetary policy. We then turn to consideration of its performance in terms of the usual policy objective function based on expected quadratic loss. Comparing its welfare loss to that of the optimal policy, we find substantially poorer performance of the $k$-percent rule. Thus Friedman's rule appears unsatisfactory in this standard model incorporating monopolistic competition and price stickiness.

\section{The Model}

We use the standard log-linearized New Keynesian model as the analytical framework, see e.g., Clarida, Gali, and Gertler (1999) for details and references to the original nonlinear models that lead to this linearization. The model contains two behavioral equations of the private sector:

$$
x_{t}=-\varphi\left(i_{t}-E_{t}^{*} \pi_{t+1}\right)+E_{t}^{*} x_{t+1}+g_{t},
$$

is the 'IS' curve derived from the Euler equation for consumer optimization and

$$
\pi_{t}=\lambda x_{t}+\beta E_{t}^{*} \pi_{t+1}+u_{t}
$$

${ }^{2}$ (Evans and Honkapohja, 2003a) indicate how many of these problems can be treated. 
is the price setting rule for the monopolistically competitive firms. Here $x_{t}$ and $\pi_{t}$ denote the output gap and inflation for period $t$, respectively. $i_{t}$ is the nominal interest rate. $E_{t}^{*} x_{t+1}$ and $E_{t}^{*} \pi_{t+1}$ denote the private sector expectations of the output gap and inflation next period. Since our focus is on learning behavior, these expectations need not be rational ( $E_{t}$ without $*$ denotes RE). The parameters $\varphi$ and $\lambda$ are positive and $\beta$ is the discount factor of the firms so that $0<\beta<1$.

For simplicity, the shocks $g_{t}$ and $u_{t}$ are assumed to be observable random shocks, where:

$$
\left(\begin{array}{l}
g_{t} \\
u_{t}
\end{array}\right)=V\left(\begin{array}{l}
g_{t-1} \\
u_{t-1}
\end{array}\right)+\left(\begin{array}{c}
\tilde{g}_{t} \\
\tilde{u}_{t}
\end{array}\right),
$$

and where:

$$
V=\left(\begin{array}{cc}
\mu & 0 \\
0 & \rho
\end{array}\right)
$$

$0<|\mu|<1,0<|\rho|<1$ and $\tilde{g}_{t} \sim \operatorname{iid}\left(0, \sigma_{g}^{2}\right), \tilde{u}_{t} \sim \operatorname{iid}\left(0, \sigma_{u}^{2}\right)$ are independent white noise. $g_{t}$ represents shocks to government purchases and as well as to potential output. $u_{t}$ represents any cost push shocks to marginal costs other than those entering through $x_{t}$. To simplify the analysis, we also assume throughout the paper that $\mu$ and $\rho$ are known (if not, these parameters could be made subject to learning).

It remains to specify how monetary policy is conducted. ${ }^{3}$ There are two natural possibilities for the choice of the monetary instrument: the interest rate and the money supply. We consider each in turn, starting with the former.

\section{Optimal Interest-Rate Setting}

We consider an interest-rate policy that is derived explicitly to maximize a policy objective function. This is frequently taken to be of the quadratic loss form, i.e.

$$
E_{t} \sum_{s=0}^{\infty} \beta^{s}\left[\left(\pi_{t+s}-\bar{\pi}\right)^{2}+\alpha x_{t+s}^{2}\right],
$$

where $\bar{\pi}$ is the inflation target. This type of optimal policy is often called 'flexible inflation targeting' in the current literature, see e.g., Svensson (1999, 2003). $\alpha$ is the relative weight on the output target and strict inflation targeting would be the case $\alpha=0$. The policymaker is assumed to have the same discount factor $\beta$ as the private sector. ${ }^{4}$ We remark that the presence of the two shocks $g_{t}$ and $u_{t}$ makes the problem of policy optimization non-trivial, since policy has only a

\footnotetext{
${ }^{3}$ As is common, we leave hidden the government budget constraint and the equation for the evolution of government debt. This is acceptable provided fiscal policy appropriately accommodates the consequences of monetary policy for the government budget constraint. See for example, Chadha and Nolan (2003). The interaction of monetary and fiscal policy can be important for the stability of equilibria under learning, see Evans and Honkapohja (2002b) and McCallum (2002).

${ }^{4}$ It is well known that the objective function (4) can be interpreted as a quadratic approximation to the utility function of the representative agent.
} 
single instrument, the interest rate or the money supply, under its control. The $u_{t}$ shock is particularly troublesome as it leads to a trade-off between the variability of the output gap and the variability of inflation.

The literature on optimal policy distinguishes between optimal policy under commitment and discretion, e.g. compare Evans and Honkapohja (2002a) and Evans and Honkapohja (2003b). Under commitment the policymaker can do better because commitment can have effects on private expectations beyond those achieved under discretion. Solving the problem of minimizing (4), subject to (2) holding in every period, and assuming RE leads to a series of first order conditions for the optimal dynamic policy. This policy exhibits time inconsistency, in the sense that policymakers would have an incentive to deviate from the policy in the future. However, this policy performs better than discretionary policy.

Assuming that the policy has been initiated at some point in the past and setting $\bar{\pi}=0$ without loss of generality, the first-order condition specifies:

$$
\lambda \pi_{t}+\alpha\left(x_{t}-x_{t-1}\right)=0
$$

in every period. ${ }^{5}$ In contrast the corresponding policy under discretion specifies $\lambda \pi_{t}+\alpha x_{t}=0$. We will focus on the commitment case, which delivers superior performance.

Condition (5) for optimal policy with commitment is not a complete specification of monetary policy, since one must still determine an $i_{t}$ rule (also called a 'reaction function') that implements the policy. It turns out that a number of interest-rate rules are consistent with the model (1)-(2), the optimality condition (5), and rational expectations. Some of the ways of implementing 'optimal' monetary policy make the economy vulnerable to either indeterminacy or instability under learning or both, while other implementations are robust to these difficulties. For an overview see Evans and Honkapohja (2003a).

Expectations-based optimal rules are advocated in Evans and Honkapohja (2002a), who argue that observable private expectations should be appropriately incorporated into the interest-rate rule. If this is done, it can be shown that the REE will be stable under learning and thus optimal policy can be successfully implemented. The desired rule is obtained by combining the IS curve (1), the price setting equation (2) and the first-order optimality condition (5), treating the private expectations as given. Eliminating $x_{t}$ and $\pi_{t}$ form these equations, but not imposing the rational expectations assumption, leads to an interest-rate equation:

$$
i_{t}=\delta_{L} x_{t-1}+\delta_{\pi} E_{t}^{*} \pi_{t+1}+\delta_{x} E_{t}^{*} x_{t+1}+\delta_{g} g_{t}+\delta_{u} u_{t}
$$

\footnotetext{
${ }^{5}$ Treating the policy as having been initiated in the past corresponds to the timeless perspective' described by Woodford (1999a, 1999b).
} 
under commitment with coefficients:

$$
\begin{aligned}
& \delta_{L}=\frac{-\alpha}{\varphi\left(\alpha+\lambda^{2}\right)}, \\
& \delta_{\pi}=1+\frac{\lambda \beta}{\varphi\left(\alpha+\lambda^{2}\right)}, \delta_{x}=\varphi^{-1}, \\
& \delta_{g}=\varphi^{-1}, \delta_{u}=\frac{\lambda}{\varphi\left(\alpha+\lambda^{2}\right)} .
\end{aligned}
$$

Given the interest-rate rule (6) we can obtain the reduced form of the model and study its properties. The reduced form is:

$$
\begin{aligned}
\left(\begin{array}{c}
x_{t} \\
\pi_{t}
\end{array}\right)= & \left(\begin{array}{cc}
0 & -\frac{\lambda \beta}{\alpha+\lambda^{2}} \\
0 & \frac{\alpha \beta}{\alpha+\lambda^{2}}
\end{array}\right)\left(\begin{array}{c}
E_{t}^{*} x_{t+1} \\
E_{t}^{*} \pi_{t+1}
\end{array}\right) \\
& +\left(\begin{array}{cc}
\frac{\alpha}{\alpha+\lambda^{2}} & 0 \\
\frac{\alpha \lambda}{\alpha+\lambda^{2}} & 0
\end{array}\right)\left(\begin{array}{c}
x_{t-1} \\
\pi_{t-1}
\end{array}\right)+\left(\begin{array}{cc}
0 & -\frac{\lambda}{\alpha+\lambda^{2}} \\
0 & \frac{\alpha}{\alpha+\lambda^{2}}
\end{array}\right)\left(\begin{array}{l}
g_{t} \\
u_{t}
\end{array}\right) .
\end{aligned}
$$

Defining:

$$
y_{t}=\left(\begin{array}{c}
x_{t} \\
\pi_{t}
\end{array}\right) \quad \text { and } \quad v_{t}=\left(\begin{array}{l}
g_{t} \\
u_{t}
\end{array}\right),
$$

the reduced form (7) can be written as:

$$
y_{t}=M E_{t}^{*} y_{t+1}+N y_{t-1}+P v_{t}
$$

for appropriate matrices $M, N$ and $P$.

We are interested in the determinacy (uniqueness) of the stationary RE solution and the stability under learning of the REE of interest. The next section outlines these concepts and the methodology for assessing determinacy and stability under learning for multivariate models such as (7).

\section{Methodology: determinacy and stability under learning}

\section{(i) Determinacy}

The first issue of concern is whether under rational expectations the system possesses a unique stationary REE, in which case the model is said to be 'determinate.' If instead the model is 'indeterminate,' there exist multiple stationary solutions and these will include undesirable 'sunspot solutions', i.e. REE depending on extraneous random variables that influence the economy solely through the expectations of the agents. ${ }^{6}$

\footnotetext{
${ }^{6}$ The possibility of interest rate rules leading to indeterminacy was demonstrated in Bernanke and Woodford (1997), Woodford (1999b) and Svensson and Woodford (2003) and this issue was further investigated in Bullard and Mitra (2002) and Evans and Honkapohja (2002a, 2003b).
} 
Formally, in the determinate case the unique stationary solution for the model (8) takes the 'minimal state variable' (or MSV) form:

$$
y_{t}=a+b y_{t-1}+c v_{t},
$$

for appropriate values $(a, b, c)=(0, \bar{b}, \bar{c})$. In the indeterminate case there are multiple stationary solutions of this form, as well as non-MSV REE. The general methodology for ascertaining determinacy is given in the Appendix to Chapter 10 of Evans and Honkapohja (2001). The procedure is to rewrite the model in first-order form and compare the number of non-predetermined variables with the number of roots of the forward looking matrix that lie inside the unit circle.

For reduced form (7) we make use of the fact that the second column of $N$ is zero. Writing $M=\left(\begin{array}{ll}m_{11} & m_{12} \\ m_{21} & m_{22}\end{array}\right)$ and $N=\left(\begin{array}{ll}n_{11} & 0 \\ n_{21} & 0\end{array}\right)$, assuming rational expectations, introducing the new variable $x_{t}^{L} \equiv x_{t-1}$, and noting that for any random variable $z_{t+1}$ we have $E_{t} z_{t+1}=z_{t+1}+\varepsilon_{t+1}^{z}$ where $E_{t} \varepsilon_{t+1}^{z}=0$, we can rewrite (8) as:

$$
\left(\begin{array}{ccc}
1 & 0 & -n_{11} \\
0 & 1 & -n_{12} \\
1 & 0 & 0
\end{array}\right)\left(\begin{array}{c}
x_{t} \\
\pi_{t} \\
x_{t}^{L}
\end{array}\right)=\left(\begin{array}{ccc}
m_{11} & m_{12} & 0 \\
m_{21} & m_{22} & 0 \\
0 & 0 & 1
\end{array}\right)\left(\begin{array}{l}
x_{t+1} \\
\pi_{t+1} \\
x_{t+1}^{L}
\end{array}\right)+\text { other }
$$

where 'other' includes terms that are not relevant in assessing determinacy. Assuming $n_{11} \neq 0$ this can be rewritten as:

$$
\left(\begin{array}{c}
x_{t} \\
\pi_{t} \\
x_{t}^{L}
\end{array}\right)=J\left(\begin{array}{c}
x_{t+1} \\
\pi_{t+1} \\
x_{t+1}^{L}
\end{array}\right)+\text { other }
$$

where:

$$
J=\left(\begin{array}{ccc}
1 & 0 & -n_{11} \\
0 & 1 & -n_{12} \\
1 & 0 & 0
\end{array}\right)^{-1}\left(\begin{array}{ccc}
m_{11} & m_{12} & 0 \\
m_{21} & m_{22} & 0 \\
0 & 0 & 1
\end{array}\right)
$$

Because this model has one predetermined variable, i.e. $x_{t}^{L}$, the condition for determinacy is that exactly two eigenvalues of $J$ lie inside the unit circle and one eigenvalue outside. If one or no roots lie inside the unit circle (with the other roots outside), the model is indeterminate.

\section{(ii) Stability under learning}

The second basic issue for models of the form (8) concerns stability under adaptive learning. If private agents follow an adaptive learning rule, will the RE solution of interest be stable, i.e. reached asymptotically by the learning process? If not, the REE is unlikely to be reached because the specified policy is potentially destabilizing. ${ }^{7}$ As is usual in the literature, we specifically model learning by agents as taking the form of least squares estimates of parameters that are updated recursively as new data are generated.

${ }^{7}$ This is the focus of the papers by Bullard and Mitra (2001, 2002), Evans and Honkapohja (2002a, 2003b) and others. 
To examine stability under least squares learning we treat (9) as the Perceived Law of Motion (PLM) of the agents, i.e. as the form of their econometric model, and assume that agents estimate its coefficients $a, b, c$ using the available data. (9) is a vector autoregression (VAR) with exogenous variables $v_{t}$, and the estimates $\left(a_{t}, b_{t}, c_{t}\right)$ are updated at each point in time by recursive least squares. Using these estimates, private agents form expectations according to $E_{t}^{*} y_{t+1}=$ $a_{t}+b_{t}\left(a_{t}+b_{t} y_{t-1}+c_{t} v_{t}\right)+c_{t} V v_{t}$ (where we are assuming for convenience that $V$ is known), and $y_{t}$ is generated according to (8). Then at the beginning of $t+1$ agents use the last data point to update their parameter estimates to $\left(a_{t+1}, b_{t+1}, c_{t+1}\right)$, and the process continues. The question is whether over time $\left(a_{t}, b_{t}, c_{t}\right) \rightarrow(0, \bar{b}, \bar{c})$. It can be shown that the E-stability principle gives the conditions for local convergence of least squares learning. In what follows, we exploit this connection between convergence of learning dynamics and E-stability. ${ }^{8}$

To define E-stability we compute the mapping from the PLM to the Actual Law of Motion (ALM) as follows. The expectations corresponding to (9), for given parameter values $(a, b, c)$, are given by:

$$
E_{t}^{*} y_{t+1}=a+b\left(a+b y_{t-1}+c v_{t}\right)+c V v_{t},
$$

where we are treating the information set available to the agents, when forming expectations, as including $v_{t}$ and $y_{t-1}$ but not $y_{t}$. (Alternative information assumptions are straightforward to consider.) This leads to the mapping from PLM to ALM given by:

$$
T(a, b, c)=\left(M(I+b) a, M b^{2}+N, M(b c+c V)+P\right) .
$$

E-stability is determined by local asymptotic stability of $\operatorname{REE}(0, \bar{b}, \bar{c})$ under the differential equation:

$$
\frac{d}{d \tau}(a, b, c)=T(a, b, c)-(a, b, c)
$$

and the E-stability conditions govern stability under least squares learning. The stability conditions can be stated in terms of the derivative matrices:

$$
\begin{aligned}
& D T_{a}=M(I+\bar{b}), \\
& D T_{b}=\bar{b}^{\prime} \otimes M+I \otimes M \bar{b}, \\
& D T_{c}=V^{\prime} \otimes M+I \otimes M \bar{b},
\end{aligned}
$$

where $\otimes$ denotes the Kronecker product and $\bar{b}$ denotes the REE value of $b$. The necessary and sufficient condition for E-stability is that all eigenvalues of $D T_{a}-I, D T_{b}-I$ and $D T_{c}-I$ have negative real parts. ${ }^{9}$

\footnotetext{
${ }^{8}$ Evans and Honkapohja (2001) provide an extensive analysis of adaptive learning and its implications in macroeconomics.

${ }^{9} \mathrm{We}$ are excluding the exceptional cases where one or more eigenvalue has zero real part.
} 


\section{Results for optimal interest-rate setting}

Monetary policy that is based on the optimal interest-rate rule (6) will lead to both determinacy and stability and learning. Evans and Honkapohja (2002a) prove the following results to this effect.

Proposition 1: Under the expectations-based reaction function (6) the REE is determinate for all structural parameter values.

It is clearly a desirable property of our proposed monetary policy rule that it does not permit the existence of other suboptimal stationary REE. However, having a determinate REE does not ensure that it is attainable under learning and we next consider this issue for the economy under the interest-rate rule (6).

Proposition 2: Under the expectations-based reaction function (6), the optimal REE is stable under learning for all structural parameter values.

We remark that the expectations-based rule (6) obeys a form of the Taylor principle since $\delta_{\pi}>1$. Partial intuition for Proposition 2 can be seen from the reduced form (7). An increase in inflation expectations leads to an increase in actual inflation that is smaller than the change in expectations since $\alpha \beta /$ $\left(\alpha+\lambda^{2}\right)<1$, where the dampening effect arises from the interest-rate reaction to changes in $E_{t}^{*} \pi_{t+1}$ and is a crucial element of the stability result. ${ }^{10}$

\section{Friedman's Money Supply Rule}

Friedman's rule stipulates that the nominal money supply is increased by a constant percentage $k$ from one period to the next. In logarithms the nominal money supply $M_{t}$ must thus satisfy:

$$
M_{t}=M+k t+w_{t},
$$

where $M$ is a constant, $k$ is the percentage increase in money supply and $w_{t}$ denotes a random noise term, which is assumed to be white noise for simplicity.

The demand for real balances is assumed to depend positively on the output gap $x_{t}$ and negatively on the nominal interest rate $i_{t}$ and a possible iid random shock $e_{t}$. The money market equilibrium or LM curve can then be written as:

$$
M+k t+w_{t}-p_{t}=\theta x_{t}-\eta^{-1} i_{t}+e_{t},
$$

where $p_{t}$ is the log of the price level. This yields the formula:

$$
i_{t}=\eta \theta x_{t}+\eta p_{t}-\eta k t-\eta M+\eta\left(e_{t}-w_{t}\right)
$$

for the nominal interest rate. Note that (18) bears a resemblance to a Taylor rule, except that $i_{t}$ now reacts on the price level rather than inflation. It can also be thought of as a form of flexible nominal income targeting in which the

\footnotetext{
${ }^{10}$ We remark that an alternative information assumption, which allows forecasts to be functions also of current endogenous variables, is sometimes used in the literature. Stability under the expectations-based reaction function continues to hold for this case.
} 
weights on real output and prices are not constrained to be equal. ${ }^{11}$ We also remark that, in contrast to (6), the interest rate under (18) responds to monetary shocks $e_{t}$ and $w_{t}$ and does not depend on the shocks in the IS and price setting equations.

Substituting (18) into the IS curve (1) leads to the expression:

$$
\begin{aligned}
x_{t}= & -\varphi \eta \theta x_{t}-\varphi \eta p_{t}+\varphi E_{t}^{*} \pi_{t+1} \\
& +\varphi \eta k t+E_{t}^{*} x_{t+1}+\varphi \eta M-\varphi \eta\left(e_{t}-w_{t}\right)+g_{t},
\end{aligned}
$$

which together with the New Phillips curve (2) and the definition of the inflation rate:

$$
p_{t}=\pi_{t}+p_{t-1}
$$

yield the model to be analyzed.

We first consider the perfect foresight steady state when there are no random shocks. It is easily computed as:

$$
x_{t}=\lambda^{-1}(1-\beta) k, \quad \pi_{t}=k \quad \text { and } \quad p_{t}=a+k t,
$$

where $a=M-\theta \lambda^{-1}(1-\beta) k$.

The next step is to write the model in deviation form from the non-stochastic steady state. Using the same notation $x_{t}, \pi_{t}$ and $p_{t}$ for the deviated variables we have the matrix form:

$$
\begin{aligned}
& \left(\begin{array}{ccc}
1+\varphi \eta \theta & 0 & \varphi \eta \\
-\lambda & 1 & 0 \\
0 & -1 & 1
\end{array}\right)\left(\begin{array}{c}
x_{t} \\
\pi_{t} \\
p_{t}
\end{array}\right) \\
= & \left(\begin{array}{ccc}
1 & \varphi & 0 \\
0 & \beta & 0 \\
0 & 0 & 0
\end{array}\right)\left(\begin{array}{l}
E_{t}^{*} x_{t+1} \\
E_{t}^{*} \pi_{t+1} \\
E_{t}^{*} p_{t+1}
\end{array}\right)+\left(\begin{array}{lll}
0 & 0 & 0 \\
0 & 0 & 0 \\
0 & 0 & 1
\end{array}\right)\left(\begin{array}{l}
x_{t-1} \\
\pi_{t-1} \\
p_{t-1}
\end{array}\right)+\left(\begin{array}{l}
\bar{g}_{t} \\
u_{t} \\
0
\end{array}\right),
\end{aligned}
$$

where $\bar{g}_{t}=g_{t}-\varphi \eta\left(e_{t}-w_{t}\right)$. The inverse of the matrix on the left hand side of (21) is:

$$
\left(\begin{array}{ccc}
r & -\varphi \eta r & -\varphi \eta r \\
\lambda r & (1+\varphi \eta \theta) r \varphi \lambda \eta r & \\
\lambda r & (1+\varphi \eta \theta) r & (1+\varphi \eta \theta) r
\end{array}\right)
$$

${ }^{11}$ The case of nominal income targeting is studied in Mitra (2003). 
where $r^{-1}=1+\eta \varphi(\theta+\lambda)$, and so we get the system:

$$
\begin{aligned}
\left(\begin{array}{c}
x_{t} \\
\pi_{t} \\
p_{t}
\end{array}\right)= & \left(\begin{array}{ccc}
r & r \varphi(1-\beta \eta) & 0 \\
r \lambda & r[\lambda \varphi+\beta(1+\eta \theta \varphi)] & 0 \\
r \lambda & r[\lambda \varphi+\beta(1+\eta \theta \varphi)] & 0
\end{array}\right)\left(\begin{array}{c}
E_{t}^{*} x_{t+1} \\
E_{t}^{*} \pi_{t+1} \\
E_{t}^{*} p_{t+1}
\end{array}\right) \\
& +\left(\begin{array}{ccc}
0 & 0 & r \eta \varphi \\
0 & 0 & r \eta \lambda \varphi \\
0 & 0 & r(1+\eta \theta \varphi)
\end{array}\right)\left(\begin{array}{l}
x_{t-1} \\
\pi_{t-1} \\
p_{t-1}
\end{array}\right) \\
& +\left(\begin{array}{cc}
r & -\eta \varphi r \\
\lambda r & (1+\eta \varphi \theta) r \\
\lambda r & (1+\eta \varphi \theta) r
\end{array}\right)\left(\begin{array}{l}
\bar{g}_{t} \\
u_{t}
\end{array}\right) .
\end{aligned}
$$

Introducing the vector notation:

$$
z_{t}=\left(\begin{array}{c}
x_{t} \\
\pi_{t} \\
p_{t}
\end{array}\right)
$$

we write (22) in the general form:

$$
z_{t}=F E_{t}^{*} z_{t+1}+G z_{t-1}+H v_{t} .
$$

\section{Determinacy}

Analysis of determinacy of the model can be done using the same general methodology that was outlined in Section III under the heading Determinacy for study of the model with interest-rate setting. Examining the reduced form (22) we note that the model has one predetermined variable $p_{t-1}$. Thus we introduce a new variable $q_{t}=p_{t-1}$ and write (22) as:

$$
\begin{aligned}
& \left(\begin{array}{cccc}
1 & 0 & 0 & -r \eta \varphi \\
0 & 1 & 0 & -r \eta \lambda \varphi \\
0 & 0 & 1-r(1+\eta \theta \varphi) & \\
0 & 0 & 1 & 0
\end{array}\right)\left(\begin{array}{c}
x_{t} \\
\pi_{t} \\
p_{t} \\
q_{t}
\end{array}\right) \\
= & \left(\begin{array}{cccc}
r & r \varphi(1-\beta \eta) & 0 & 0 \\
r \lambda & r[\lambda \varphi+\beta(1+\eta \theta \varphi)] & 0 & 0 \\
r \lambda & r[\lambda \varphi+\beta(1+\eta \theta \varphi)] & 0 & 0 \\
0 & 0 & 0 & 1
\end{array}\right)\left(\begin{array}{c}
E_{t}^{*} x_{t+1} \\
E_{t}^{*} \pi_{t+1} \\
E_{t}^{*} p_{t+1} \\
E_{t}^{*} q_{t+1}
\end{array}\right)
\end{aligned}
$$


or in symbolic form:

$$
A \hat{y}_{t}=B E_{t}^{*} \hat{y}_{t+1},
$$

where $\hat{y}_{t}=\left(x_{t}, \pi_{t}, p_{t}, q_{t}\right)^{\prime}$ and the matrices $A$ and $B$ are those specified by (24). Determinacy obtains when exactly three eigenvalues of the matrix $A^{-1} B$ are inside the unit circle.

It is evident from (24) that it would be difficult to obtain general theoretical results on determinacy, and we thus examine the issue numerically. ${ }^{12}$ We use two different sets of the calibrated parameter values, respectively suggested by Woodford (1996) and McCallum and Nelson (1999). Thus consider the examples:

Calibrated examples:

$\mathbf{W}: \eta=0.053, \theta=1, \varphi=1, \lambda=0.3, \beta=0.95$.

MN: $\eta=0.090, \theta=0.930, \varphi=0.164, \lambda=0.3, \beta=0.99$.

For the shocks we assume that $\mu=\rho=0.4$ and that there are no monetary shocks. For the $\mathrm{W}$ and $\mathrm{MN}$ parameter values the eigenvalues of $A^{-1} B$ are:

$\mathrm{W}: 0,0.563,0.950$ and 1.687 ;

MN: 0, 0.843, 0.902 and 1.284.

We conclude:

Result 3: The Friedman k-percent rule leads to determinacy of equilibria.

We have expressed this as a 'result' rather than a proposition because it has been verified only for the two calibrated examples.

\section{Stability under learning}

As discussed above in Section III under the heading Stability under learning, we can focus on E-stability of the (determinate) REE in model (22) to determine the stability of the REE under adaptive learning.

We first derive convenient expressions for the REE. Since the model has only one lagged endogenous variable $p_{t-1}$ we guess that the MSV REE takes the form: ${ }^{13}$

$$
\begin{aligned}
& z_{t}=C z_{t-1}+K v_{t}, \\
& C=\left(\begin{array}{lll}
0 & 0 & c_{x} \\
0 & 0 & c_{\pi} \\
0 & 0 & c_{p}
\end{array}\right) .
\end{aligned}
$$

\footnotetext{
${ }^{12}$ The Mathematica routines are available on request from the authors.

${ }^{13}$ Note that the shocks can be written as $v_{t}$ since the monetary shocks were assumed away. 
Guessing that the REE has this form, we obtain that the REE must satisfy the equations:

$$
\begin{aligned}
& c_{x}=r c_{x} c_{\pi}+r \varphi(1-\beta \eta) c_{\pi} c_{p}+r \eta \varphi \\
& c_{\pi}=r \lambda c_{x} c_{\pi}+r[\lambda \varphi+\beta(1+\eta \theta \varphi)] c_{\pi} c_{p}+r \eta \lambda \varphi, \\
& c_{p}=r \lambda c_{x} c_{\pi}+r[\lambda \varphi+\beta(1+\eta \theta \varphi)] c_{\pi} c_{p}+r(1+\eta \theta \varphi)
\end{aligned}
$$

and

$$
[I-(I \otimes F C)-(V \otimes F)] \operatorname{vec} K=\operatorname{vec} H,
$$

where vec refers to vectorization of the matrix. For the calibrated examples above the stationary RE solution is for W calibration:

$$
\begin{aligned}
\bar{c}_{x} & =0.592837, \bar{c}_{\pi}=0.407163, \bar{c}_{p}=0.592837, \\
\bar{K} & =\left(\begin{array}{rr}
1.17984 & -0.76523 \\
0.35156 & 0.76523 \\
0.35156 & 0.76523
\end{array}\right)
\end{aligned}
$$

and for MN calibration:

$$
\begin{aligned}
& \bar{c}_{x}=0.169118, \bar{c}_{\pi}=-0.221386, \bar{c}_{p}=0.778614, \\
& \bar{K}=\left(\begin{array}{rr}
1.49239 & -0.28026 \\
0.54389 & 0.54389 \\
0.54389 & 0.54389
\end{array}\right) .
\end{aligned}
$$

To study E-stability one postulates that the agents in the economy have perceived law of motion (PLM) that takes the form:

$$
z_{t}=a+C z_{t-1}+K v_{t},
$$

where the parameter vector $a$ and the matrices $C$ and $K$ are in general not equal to the REE values. Agents forecast using the PLM, which leads to forecast functions: ${ }^{14}$

$$
E_{t}^{*} z_{t+1}=(I+C) a+C^{2} z_{t-1}+(C K+K V) v_{t} .
$$

This forecast function is substituted into (23), which yields the temporary equilibrium given the forecasts or the actual law of motion (ALM):

$$
z_{t}=F(I+C) a+\left(F C^{2}+G\right) z_{t-1}+[F(C K+K V)+H] v_{t} .
$$

The E-stability condition is that all eigenvalues of the matrices:

$$
F(I+\bar{C}), \bar{C}^{\prime} \otimes F+I \otimes F \bar{C} \text { and } I \otimes F \bar{C}+V \otimes F
$$

have real parts less than one. $\otimes$ again denotes the Kronecker product.

\footnotetext{
${ }^{14}$ As was done earlier, it is assumed that the agents do not see the current value of $z_{t}$ when they form expectations. This is a standard assumption in the literature.
} 
Analytical results on E-stability cannot be obtained in view of the complexity of the model. We thus evaluated numerically the eigenvalues of these matrices using the calibrated examples specified above. For $\mathrm{W}$ calibration the eigenvalues of $F(I+\bar{C})$ are $-7.85046 \times 10^{-17}, 0.576656$ and 0.913702 . The eigenvalues of $\bar{C}^{\prime} \otimes F+I \otimes F \bar{C}$ are $-0.661714,0.307057 \pm 0.0595477 i, \quad 3.44306 \times 10^{-16}$ and four eigenvalues equal to zero. The eigenvalues of $I \otimes F \bar{C}+V \otimes F$ are 0.21117 , -0.01206 and 0 where each of these is a double root. For MN calibration the eigenvalues of $F(I+\bar{C})$ are 0 , and $0.868719 \pm 0.0490926 i$. The eigenvalues of $\bar{C}^{\prime} \otimes F+I \otimes F \bar{C}$ are -0.27847 (twice), $0.645542 \pm 0.0717222 i$, and five eigenvalues equal to zero. The eigenvalues of $I \otimes F \bar{C}+V \otimes F$ are $0.27627,0.251457$ and 0 where each of these is a double root.

We conclude:

Result 4: Under the Friedman $k$-percent rule the REE is stable under learning.

Results 3 and 4 might seem surprising since for calibrated parameter values the coefficients of $x_{t}$ and $p_{t}$ in the interest rate relation (18) are small. However, note that the interest rate in (18) feeds back on the price level and not the inflation rate and it is this feature that generates both determinacy and stability under learning. Numerical investigation indicates that as $\eta \downarrow 0$ then the economy approaches the borderline to indeterminacy and instability. This corresponds to the case in which money demand is infinitely interest-rate elastic. In contrast, when $\eta$ is large, determinacy and E-stability are easily satisfied.

\section{Welfare comparison}

We now compare the performance of the Friedman rule to optimal policy under commitment. (The Appendix outlines the method of calculating welfare losses.) In this comparison we assume that the monetary shocks are both zero. Monetary shocks would feed into the behavior of output gap and inflation through the term $\bar{g}_{t}$ in (21) under the Friedman rule. In contrast, monetary shocks play no role under an interest-rate policy, since both money demand and supply are then endogenous but do not affect the welfare loss.

We need to fix some additional parameters for this computation and choose $\alpha=0.1, \sigma_{g}^{2}=1$ and $\sigma_{u}^{2}=0.5^{2}$. For the two calibrations we get the following values for the loss function under the Friedman rule (denoted as $W_{F r}$ ) and under the optimal expectations-based rule with commitment (denoted as $W_{E B}$ ):

$$
\begin{aligned}
\mathbf{W}: W_{F r} & =0.423826, W_{E B}=0.172182 \\
\mathbf{M N}: W_{F r} & =0.830019, W_{E B}=0.169408 .
\end{aligned}
$$

Compared to the optimal policy the Friedman rule delivers quite poor welfare results, at least for these calibrations. ${ }^{15}$

\footnotetext{
${ }^{15}$ In fact even the optimal discretionary policy does much better than the Friedman rule for these parameter settings, yielding welfare losses of $W=0.205592$ and $W=0.20999$ for the $\mathbf{W}$ and MN calibrations, respectively.
} 
These comparisons give the welfare for the REE, i.e. they can be viewed as the welfare achieved asymptotically. It would also be of interest to consider the speed of convergence of the learning under the two monetary policy rules, which would have transitional effects on welfare. It is known that the speed of convergence is governed by the eigenvalues of the $T$-map used to define Estability. In particular, the asymptotic speed of convergence is controlled by the largest real part of the eigenvalues of the $T$-map, see Chapter 7 of Evans and Honkapohja (2001): convergence is slower the closer the real part of this dominant eigenvalue is to one. For the particular calibrations used above we have the following values:

$$
\begin{aligned}
\text { W: } \operatorname{Eig}_{F r} & =0.914, \operatorname{Eig}_{E B}=0.203 \\
\text { MN: } \operatorname{Eig}_{F r} & =0.869, \operatorname{Eig}_{\bar{E} B}=0.209 .
\end{aligned}
$$

Thus for these calibrations convergence to REE under the expectations-based rule would be much faster than under the Friedman rule. These results are, however, sensitive on parameter values. In particular, for the expectations-based rule the value of Eig increases as $\alpha$ is increased. Similarly, for the Friedman rule the value of Eig depends sensitively on $\eta$ : higher values of $\eta$ lead to faster convergence.

\section{CONCLUding REMARKS}

We began by reviewing the results on optimal interest-rate policy, and presented an implementation that achieves both determinacy and stability under learning of the optimal REE. This optimal policy rule relies on strong feedback from the expectations of private agents, and also requires knowledge of key structural parameters for the economy. Clearly, these are strong informational requirements. However, simpler open-loop interest-rate rules, for example those depending only on exogenous shocks, fail to be stable under learning and also suffer from indeterminacy problems.

Friedman's money supply rule has a major advantage in terms of simplicity. We first examined whether the Friedman $k$-percent money supply rule leads to determinacy of equilibria. Due to the complexity of the model, analytical results were not obtainable. However, numerical analysis indicated that Friedman's rule does lead to determinate equilibria. We then considered whether the unique stationary REE is stable under learning. Here we employed the concept of E-stability which is known to provide necessary and sufficient conditions for convergence of least squares learning rules. Again, numerical analysis showed that Friedman's money supply rule delivers an REE that is stable under learning.

Finally, we studied the performance of Friedman's rule in terms of the quadratic objective function that can approximate the welfare loss of the economy. In both calibrations of the model, Friedman's rule leads to high welfare losses relative to those that are attained when monetary policy is conducted in terms of the optimal interest-rate rule. 
We conclude that, while Friedman's money supply rule performs well in terms of determinacy and stability under learning, its performance is relatively poor in terms of welfare loss. According to these results, the choice of the monetary instrument presents a dilemma. If a simple open loop policy is desired, the money supply provides a superior instrument relative to the interest rate since the latter fails the basic tests of determinacy and learnability. Yet in terms of welfare loss, an open loop money supply policy delivers poor results. There may exist simple money supply feedback policies that are much better in terms of attained welfare, but whether they would pass the basic tests of determinacy and learnability is a question that would need to be explicitly examined.

Another way to extend the analysis would be to incorporate explicitly structural parameter uncertainty into the calculations. The expectations-based rule requires knowledge of structural parameters. We show elsewhere that the policymaker could obtain consistent estimates of the required structural parameters even when private agents are following adaptive learning rules; see Evans and Honkapohja (2003a). However, at any moment of time policymakers face uncertainty about these parameters and one could numerically investigate the welfare implications of using a version of the expectations-based rule that allows for this uncertainty.

\section{ACKNOWLEDGEMENTS}

The authors thank the referees for their comments. Financial support from the US National Science Foundation, the Academy of Finland, Yrjö Jahnsson Foundation, Bank of Finland and Nokia Group is gratefully acknowledged. The views expressed are those of the authors and do not necessarily reflect the views of the Bank of Finland.

\section{APPENDIX}

\section{Welfare computation}

We calculate the expected welfare loss of the stationary REE, which is $1 /(1-\beta)$ times

$$
W=E\left(\alpha x_{t}^{2}+\pi_{t}^{2}\right) .
$$

In the case of the interest-rate rule (6) the REE solution $y_{t}=\bar{b} y_{t-1}+\bar{c} v_{t}$ can be written as:

$$
\left(\begin{array}{l}
y_{t} \\
v_{t}
\end{array}\right)=\left(\begin{array}{cc}
\bar{b} & \bar{c} V \\
0 & V
\end{array}\right)\left(\begin{array}{l}
y_{t-1} \\
v_{t-1}
\end{array}\right)+\left(\begin{array}{c}
\bar{c} \\
I
\end{array}\right) \tilde{v}_{t},
$$

where $\tilde{v}_{t}=\left(\tilde{g}_{t}, \tilde{u}_{t}\right)^{\prime}$ and $\bar{b}$ and $\bar{c}$ are the REE values under the specified interestrate rule, or:

$$
\zeta_{t}=R \zeta_{t-1}+S \tilde{v}_{t}
$$

where $\zeta_{t}^{\prime}=\left(y_{t}^{\prime}, v_{t}^{\prime}\right)$. Letting $\Sigma=\operatorname{Var}\left(\tilde{v}_{t}\right)$ denote the covariance matrix of the shocks $\tilde{v}_{t}$, the stationary covariance matrix for $\xi_{t}$ satisfies: 


$$
\operatorname{Var}\left(\zeta_{t}\right)=R \operatorname{Var}\left(\zeta_{t}\right) R^{\prime}+S \Sigma S^{\prime}
$$

or in vectorized form:

$$
\operatorname{vec}\left(\operatorname{Var}\left(\zeta_{t}\right)\right)=[I-R \otimes R]^{-1} \operatorname{vec}\left(S \Sigma S^{\prime}\right) .
$$

The variance of output gap and inflation can be read off from (27).

In the case of the money supply rule (17) we instead use the MSV solution (25) with $C=\bar{C}$ and $K=\bar{K}$, so that:

$$
\left(\begin{array}{c}
z_{t} \\
v_{t}
\end{array}\right)=\left(\begin{array}{cc}
\bar{C} & \bar{K} V \\
0 & V
\end{array}\right)\left(\begin{array}{c}
z_{t-1} \\
v_{t-1}
\end{array}\right)+\left(\begin{array}{c}
\bar{K} \\
I
\end{array}\right) \tilde{v}_{t}
$$

and $\hat{\zeta}_{t}^{\prime}=\left(z_{t}^{\prime}, v_{t}^{\prime}\right)$ is used in place of $\zeta_{t}^{\prime}$ in the computations.

\section{REFERENCES}

Altug, S., Chadha, J. S. and Nolan, C. (eds.) (2003). Dynamic Macroeconomic Theory. Cambridge UK: Cambridge University Press, forthcoming.

BERNANKE, B. and WOODFORD, M. (1997). Inflation forecasts and monetary policy. Journal of Money, Credit, and Banking, 24, pp. 653-84.

Bullard, J. and Mitra, K. (2001). Determinacy, learnability and monetary policy inertia. Working paper (www.stls.frb.org/research/econ/bullard).

Bullard, J. and Mitra, K. (2002). Learning about monetary policy rules. Journal of Monetary Economics, 49, pp. 1105-29.

Chadha, J. S. and Nolan, C. (2003). On the interaction of monetary and fiscal policy. In Altug, Chadha and Nolan (2003).

Clarida, R., Gali, J. and Gertler, M. (1999). The science of monetary policy: a new Keynesian perspective. Journal of Economic Literature, 37, pp. 1661-707.

Evans, G. W. and HonkapohJA, S. (2001). Learning and Expectations in Macroeconomics. Princeton, New Jersey: Princeton University Press.

Evans, G. W. and HonKAPOHJa, S. (2002a). Monetary policy, expectations and commitment. Mimeo (www.valt.helsinki.fi/RAKA/seppo.hmtl, and http://darkwing.uoregon.edu/ $\sim$ gevans/).

Evans, G. W. and HonKapohja, S. (2002b). Policy interaction, learning and the fiscal theory of prices. Mimeo (www.valt.helsinki.fi/RAKA/seppo.hmtl, and http://darkwing. uoregon.edu/ $\sim$ gevans/).

Evans, G. W. and Honkapohja, S. (2003a). Adaptive learning and monetary policy design. Journal of Money, Credit and Banking. Forthcoming.

Evans, G. W. and HonkAPOHJA, S. (2003b). Expectations and the stability problem for optimal monetary policies. Review of Economic Studies. Forthcoming.

Federal Reserve BanK, K. C. (1999). New Challenges for Monetary Policy. Kansas City: Federal Reserve Bank of Kansas City.

Friedman, M. (1948). A monetary and fiscal framework for monetary stability. American Economics Review, 38, pp. 245-64.

Friedman, M. (1959). A Program for Monetary Stability. New York: Fordham University Press.

HowitT, P. (1992). Interest rate control and nonconvergence to rational expectations. Journal of Political Economy, 100, pp. 776-800.

McCAllum, B. T. (2002). The fiscal theory of the price level is not learnable. Mimeo: Carnegie Mellon University.

McCallum, B. T. and Nelson, E. (1999). Performance of operational policy rules in an estimated semi-classical model. In Taylor (1999), pp. 15-45.

MitrA, K. (2003). Desirability of nominal GDP targeting under adaptive learning. Journal of Money, Credit, and Banking, 35, pp. 197-220.

Svensson, L. E. (1999). Inflation targeting as a monetary policy rule. Journal of Monetary Economics, 43, pp. 607-54. 
Svensson, L. E. (2003). What is wrong with Taylor rules? Using judgment in monetary policy through targeting rules. Journal of Economic Literature, 41, 426-477.

Svensson, L. E. O. and WoOdFord, M. (2003). Implementing optimal policy through inflation-forecast targeting. Working paper.

Taylor, J. (ed.) (1999). Monetary Policy Rules. Chicago: University of Chicago Press.

WOODFORD, M. (1996). Control of the public debt: a requirement for price stability? Working paper, NBER WP5684.

WOODFORD, M. (1999a). Commentary: how should monetary policy be conducted in an era of price stability? In Federal Reserve Bank (1999), pp. 277-316.

WoODFord, M. (1999b). Optimal monetary policy inertia. The Manchester School, Supplement, 67, pp. $1-35$.

Date of receipt of final manuscript: 21 July 2003. 